\title{
Comparative efficacy and tolerability of treatments for adult autoimmune hepatitis: A systematic review and network meta-analysis
}

\author{
FENG-BIN LU ${ }^{1 *}$, EN-DE HU ${ }^{1 *}$, LAN-MAN XU ${ }^{1}$, YI-BING HU ${ }^{2}$, LU CHEN $^{1}$, \\ JIN-LU WU ${ }^{1}$, HUI LI ${ }^{1}$, DA-ZHI CHEN ${ }^{3}$ and YONG-PING CHEN ${ }^{1}$
}

\begin{abstract}
${ }^{1}$ Wenzhou Key Laboratory of Hepatology, Department of Infectious Diseases, Hepatology Institute of Wenzhou Medical University, The First Affiliated Hospital of Wenzhou Medical University, Wenzhou, Zhejiang 325000;

${ }^{2}$ Department of Gastroenterology, The Third Affiliated Hospital of Wenzhou Medical University, Jinhua, Zhejiang 321000; ${ }^{3}$ State Key Laboratory of Infectious Diseases, Medicine School of Zhejiang University, Hangzhou, Zhejiang 310003, P.R. China
\end{abstract}

Received September 30, 2017; Accepted December 6, 2017

DOI: $10.3892 /$ etm.2018.6063

\begin{abstract}
The most suitable treatment regimen for autoimmune hepatitis (AIH) in adults remains unknown and requires further investigation. The current study therefore aimed to integrate evidence to provide hierarchies of the comparative efficacies of treatments measured by clinical and biochemical remission. A Bayesian-framework network meta-analysis of randomized controlled trials (RCTs) was preformed to compare eight treatments for AIH. Eligible RCTs were identified by searching Embase, Pubmed and the Cochrane Library for publications between 1966 and April 2017. All outcomes were independently extracted from the included studies by two authors. A total of six RCTs were subsequently included in the current study. The network of comparisons on remission indicated that patients treated with prednisone
\end{abstract}

Correspondence to: Professor Yong-Ping Chen, Wenzhou Key Laboratory of Hepatology, Department of Infectious Diseases, Hepatology Institute of Wenzhou Medical University, The First Affiliated Hospital of Wenzhou Medical University, 2 Fuxue Road, Wenzhou, Zhejiang 325000, P.R. China

E-mail: 13505777281@163.com

Dr Da-Zhi Chen, State Key Laboratory of Infectious Diseases, Medicine School of Zhejiang University, 79 Qingchun Road, Hangzhou, Zhejiang 310003, P.R. China

E-mail: dazhichen@126.com

*Contributed equally

Abbreviations: AIH, autoimmune hepatitis; pred, prednisone; AZA, azathioprine; pred-T, prednisone in titrated doses given on alternate days; bude + AZA, budesonide + azathioprine; UDCA, ursodeoxycholic acid; CsA, cyclosporine-A; RCTs, randomized controlled trials

Key words: autoimmune liver disease, liver, immunosuppression, meta-analyses (pred) experienced significantly increased rates of remission compared with those treated with azathioprine [AZA; odds ratio (OR), 0.21; 95\% confidence interval (CI), 0.06-0.71] and budesonide (bude) + AZA significantly increased remission compared with placebo treatment (OR, 36.66; 95\% CI, 1.40-962.49) or AZA (OR, 10.30; 95\% CI, 1.50-70.70). Based on the cumulative ranking probabilities, bude + AZA (89.4) was ranked first, pred (69.1) was ranked second, pred + AZA (63.2) was ranked third and placebo (7.8) treatment was ranked last. Bude + AZA may be the most appropriate candidate for the treatment of non-cirrhotic patients. However, bude + AZA as frontline therapy for AIH requires more large-scale studies with a longer duration of follow-up histology and a focus on dose-response. Additionally, development of other prospective treatments, which may be used as alternative therapy or first line therapy, and their subsequent evaluation in clinical RCTs is required.

\section{Introduction}

Autoimmune hepatitis (AIH) manifests as inflammation of the liver with nonspecific symptoms, including fatigue, jaundice and arthralgia, and some patients may develop cirrhosis, which results in mortality due to liver failure or the need for liver transplantation (1). AIH may occur at all ages and is diagnosed more often in females, and it is increasingly recognized as a global disease (2). Prednisone (pred) treatment or pred plus azathioprine (AZA) treatment has been used as the conventional treatment for AIH for $>40$ years (3). However, some patients with AIH do not respond optimally to the standard treatment and those who do respond may experience strong side effects, including infection and diabetes mellitus, or a relapse following drug withdrawal (4). Thus, development of a novel alternative treatment for AIH is required.

Several medications, including cyclosporine-A (CsA), tacrolimus, mycophenolate mofetil, budesonide and ursodeoxycholic acid (UDCA) are emerging as alternative frontline therapies for patients with AIH (5-15). CsA, budesonide (bude) and UDCA have been evaluated by randomized clinical 
trials (RCTs), and it has been demonstrated that they are just as effective or more effective than traditional treatment regimens $(7,14,15)$. Other advances in the pharmacotherapy of AIH have been derived primarily from observational studies $(5,8)$. Due to the low incidence of the disease in the general populations (mean annual incidence in white northern Europeans per 100,000 was $0.85-1.9$ for AIH; point prevalence per 100,000 was 10.7-16.9 for AIH) $(16,17)$, RCTs on the treatment of AIH are sparse and the number of patients included in RCTs is limited, leading to some potential interferences on conclusions. Therefore, the most effective treatment for AIH remains unclear.

The present study aimed to compare the efficacy of eight treatments, including pred, pred + AZA, pred in titrated doses given on alternate days (pred-T), placebo, AZA, bude + AZA, UDCA + pred and CsA for adult patients with AIH. These eight treatments were compared from six RCTs by network meta-analysis (NMA), which calculates the relative effects for all treatments, including treatments that have not been compared one by one, in the evidence network in one simultaneous analysis (18). The aim was to provide hierarchies of the comparative clinical and biochemical remission for eight treatments.

\section{Materials and methods}

Search methods. Embase (www.embase.com), Pubmed (www. ncbi.nlm.nih.gov/pubmed) and the Cochrane Library (www. cochranelibrary.com) databases were searched for publications published between 1966 and April 2017. Key words and Medical Subject Headings (the vocabulary thesaurus used for indexing articles for PubMed) terms including 'Autoimmune Hepatitides', 'Hepatitides, Autoimmune', 'Autoimmune Chronic Hepatitis', 'Autoimmune Chronic Hepatitides', 'Chronic Hepatitides, Autoimmune', 'Hepatitis, Autoimmune Chronic,' 'Autoimmune Hepatitis', 'Chronic Hepatitis, Autoimmune' and 'Hepatitides, Autoimmune Chronic', and 'treatments and/or therapies' were used during the search. The reference list for any discounted papers was also observed. Two reviewers independently made the selection of studies to include in the present study based on titles and abstracts. Any disagreement between reviewers was resolved by a further reviewer.

Inclusion and exclusion criteria. The selected studies had to fulfill the following inclusion criteria: i) Inclusion of patients with AIH; ii) inclusion of patients receiving therapeutic intervention; and iii) RCT study design that was randomized, placebo or an untreated controlled trial. Prior to the proposed the definition of AIH by the International Autoimmune Hepatitis Group in 1993, there were evolving definitions of AIH (19). Therefore, in order to check whether the search performed included all published articles that were possibly associated with the present meta-analysis, the reference lists of included papers were also scrutinized.

Exclusion criteria included: i) Articles that were not written in English, German, French or Spanish; ii) studies including traditional Chinese medicine treatment; iii) studies evaluating the efficacy of therapy for AIH in children; iv) studies evaluating the efficacy of maintenance therapy for AIH; v) studies with a follow-up of $<6$ months; and vi) case studies, case series, review studies, letters and meeting proceedings.
Data collection. A total of two review authors screened papers, removed ineligible references and contacted corresponding authors if further information was required. If the article could not be provided, the data was obtained from the author or other associated articles. Information including the first author name, journal, study date range, sample size, comparators, treatment plan, country, study design, follow-up time and three outcomes (remission, mortality rates and adverse events) were extracted for each included study. Remission, mortality rates and adverse events were used to estimate efficacy and tolerability of treatments for the network meta-analysis.

Liver biopsy was not an outcome described in all articles, therefore, clinical and biochemical remission was applied as the primary outcome measure in order to achieve the most appropriate definition of remission for the study. The definition of clinical and biochemical remission was as follows, according to previously published studies: Aspartate aminotransferase and alanine transaminase (ALT) in the normal range and absence of any clinical signs of deterioration $(14,15)$. All outcomes were extracted from the included studies and assessed at maximum follow-up.

Study quality. The Cochrane Risk of Bias Tool was used to assess the quality of included studies by two reviewers (20). The tool is based on assessing random sequence generation, allocation concealment, blinding, incomplete outcome data, selective reporting and other bias. The classification of the judgment for each domain was low risk of bias, unclear risk of bias or high risk of bias.

Data analysis. A traditional pairwise meta-analysis, which directly compared different treatments, was first performed using Stata software (version 13.0; StataCorp, College Station, TX, USA). According to the Cochrane Handbook for Systematic Reviews of Interventions Version 5.1.0, the pooled estimates of hazard ratios (HRs), odds ratios (ORs) and 95\% confidence intervals (CIs) of remission were calculated using the DerSimonian and Laird random-effects model. A $\chi^{2}$ square test and $\mathrm{I}^{2}$ test were used for testing heterogeneity among the studies. If the OR and $95 \%$ CI were not close to 1 , there was a statistical difference between the two groups. $\mathrm{P}<0.05$ indicated that the difference between groups was statistically significant.

The network meta-analysis was also performed using Stata software with the random effects models proposed by Chaimani et al (21) (downloaded from mtm.uoi.gr) according to the program. The ifplot command was used to evaluate the consistency of direct and indirect estimates. A funnel plot was used to identify possible publication bias if the number of RCTs was $>10$ (22). Posterior probabilities of outcomes were used to calculate probabilities of treatment ranking and the cumulative ranking probabilities (surface under the cumulative ranking curve; SUCRA) were used to indicate the most effective treatment.

\section{Results}

Study identification and selection. A PRISMA flow diagram of study selection is presented in Fig. 1. The search was performed on April 172017 and 9,761 references were identified. Following 


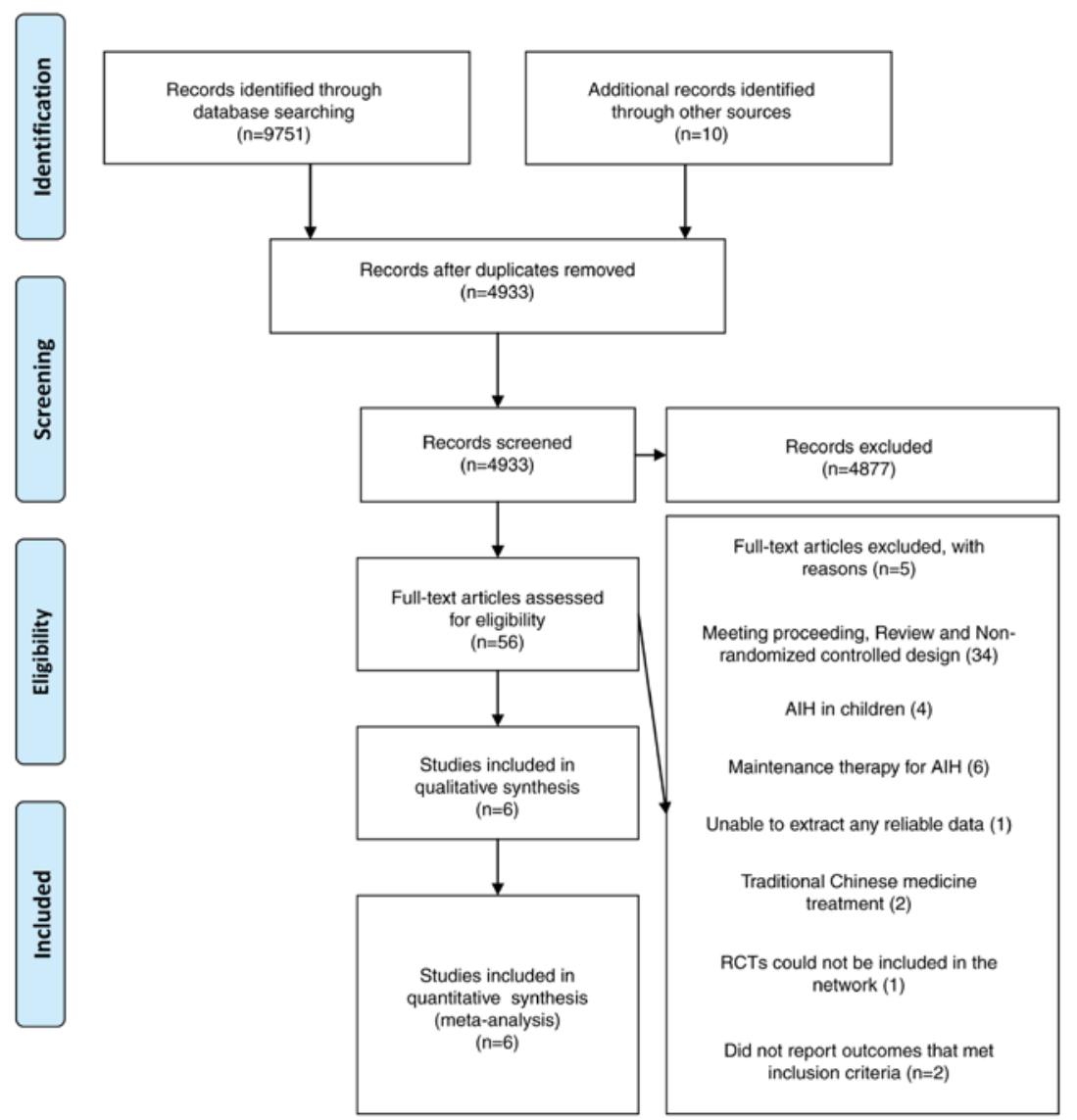

Figure 1. PRISMA flow diagram of study selection. AIH, autoimmune hepatitis; RCTs, randomized controlled trials.
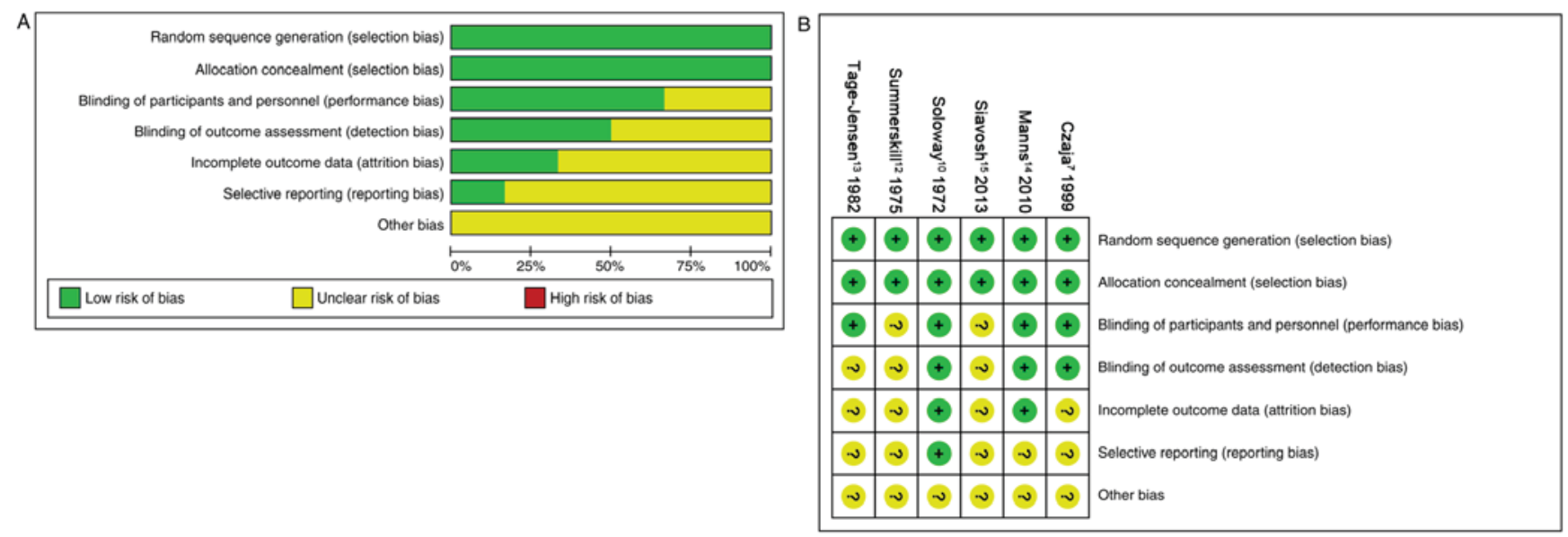

Figure 2. Risk of bias graphs. (A) Review authors' judgments on each risk of bias items presented as percentages across all included studies. (B) Review authors' judgments on each risk of bias items for each included study.

removal of 4,828 duplicate references, 4,933 records were screened. However, 4,877 articles were excluded following review of the title and abstract (e.g., studies not associated with AIH). A total of 56 studies were included in the narrative review and data from six of these studies were included in the meta-analysis. A total of 50 studies were excluded due to 34 studies not being RCT studies, four studies evaluating the efficacy of therapy for AIH in children (23-26), six studies evaluating maintenance therapy for AIH (27-31), two studies evaluating traditional Chinese medicine treatment $(32,33)$, one study being unable to extract any reliable data (34), two studies not reporting the outcome that met inclusion criteria $(9,11)$ and one study where treatment duration was indefinite and may have included the same relapsed patients with AIH as those included in another study performed by the same author (6).

Study characteristics. Table I provides a summary of the studies included in the present review. A total of six studies were included, with a total of 517 patients. All studies were RCTs directly comparing alternative treatments. A total of 


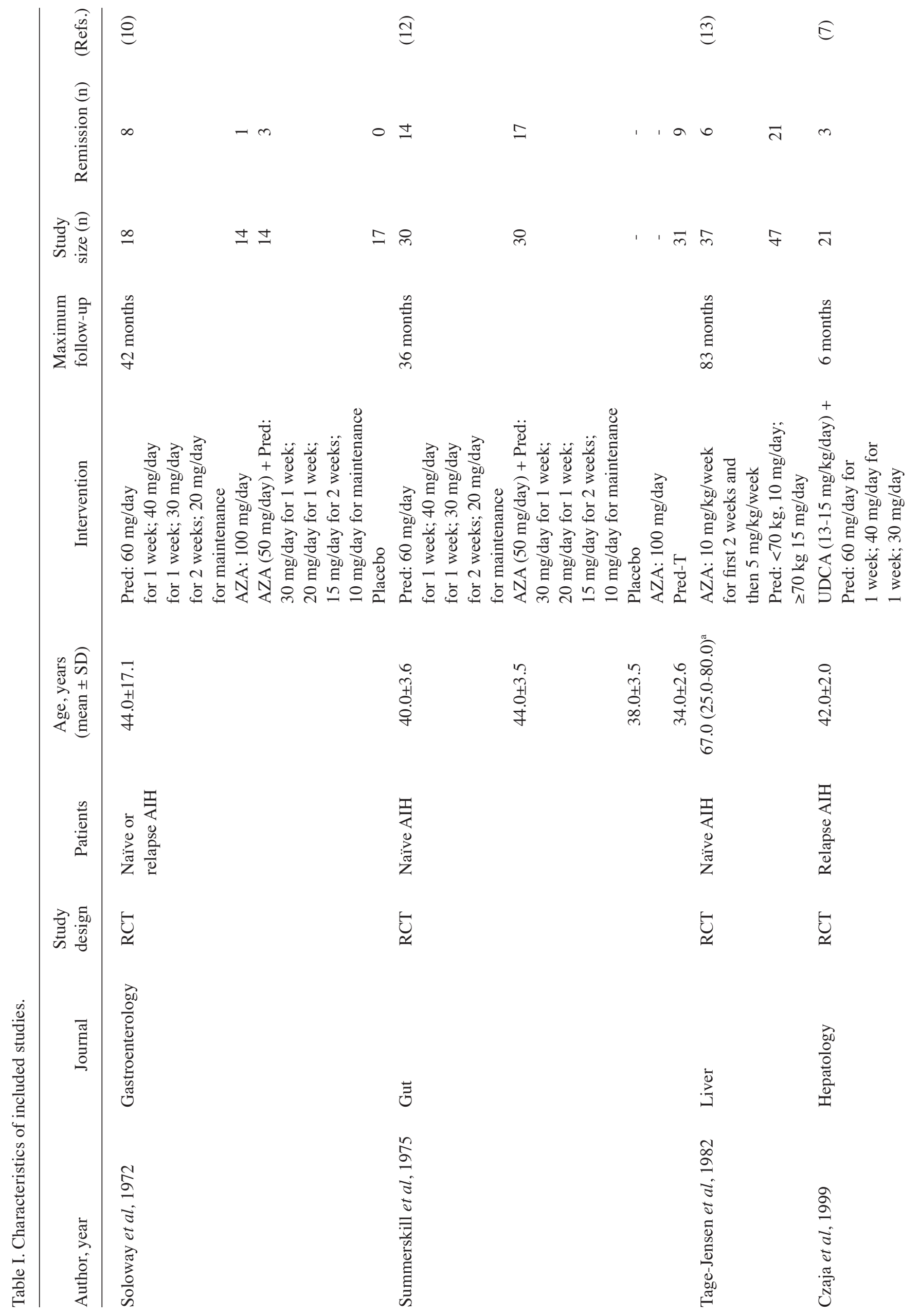




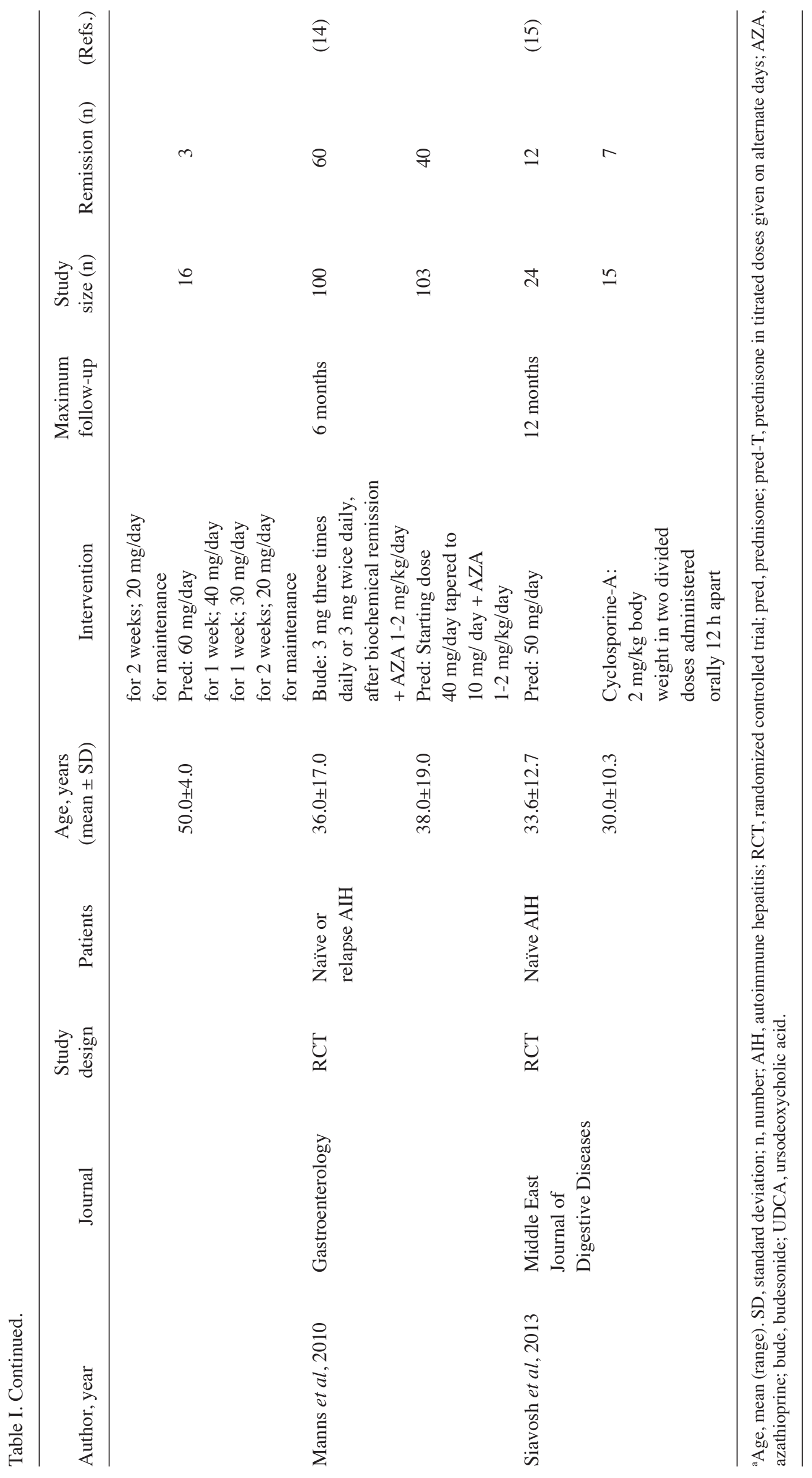


Table III. SUCRA and rank results.

\begin{tabular}{lccc}
\hline Treatment & SUCRA & PrBest & Mean rank \\
\hline Pred & 69.1 & 6.3 & 3.2 \\
Pred + AZA & 63.2 & 1.6 & 3.6 \\
Pred-T & 33.0 & 0.6 & 5.7 \\
Placebo & 7.8 & 0.5 & 7.5 \\
AZA & 18.6 & 0.0 & 6.7 \\
Bude + AZA & 89.4 & 59.2 & 1.7 \\
UDCA + pred & 56.1 & 16.4 & 4.1 \\
Cyclosporine-A & 62.8 & 15.4 & 3.6
\end{tabular}

Pred, prednisone; AZA, azathioprine; pred-T, prednisone in titrated doses given on alternate days; bude, budesonide; UDCA, ursodeoxycholic acid; SUCRA, surface under the cumulative ranking curve; PrBest, probability of being the best treatment.

four out of six studies were two-grouped studies, one was a four-grouped study and one was a five-grouped study. These studies were published between 1972 and 2013. The treatment duration was between 6 months and 6 years and the mean age of participants was 41.5 years. A total of eight treatments were included in the network meta-analysis with no treatment considered as placebo treatment. A total of six studies reported clinical and biochemical remission as the outcome.

Risk of bias in included studies. The risk of bias in all six studies is presented in Fig. 2. A total of six studies (100\%) described random sequence generation and adequate allocation concealment (100\%). A total of four studies (67\%) described blinding of participants and personnel, and three studies (50\%) had low risk of blinding of outcome assessment. A total of two studies (33\%) had a low risk of incomplete outcome data and one study (16\%) had low risk of selectively reporting results. Although some studies had dropout, the effect of intervention was not affected due to the small scale of dropout.

Effects of treatments on remission. A total of 147 patients were assigned to pred + AZA treatment, 135 to pred therapy, 100 to bude + AZA therapy, 51 to AZA therapy, 31 to pred-T therapy, 21 to UDCA + pred therapy, 17 to placebo therapy and 15 to CsA therapy.

The direct comparisons on remission were performed in Table II. The result indicated that patients treated with pred had significantly increased rates of remission compared with patients treated with AZA (OR, 0.20 ; 95\% CI, 0.08-0.53) or placebo (OR, 0.04; 95\% CI, 0.00-0.68) and patients treated with pred + AZA had a significantly increased rate of remission compared with those treated with pred-T (OR, 0.31; 95\% CI, 0.11-0.90). In addition, patients treated with bude + AZA had a significantly increased rate of remission compared with those treated with pred + AZA (OR, 2.36; 95\% CI, 1.35-4.15).

The network of comparisons on remission is presented in Fig. 3 and Table II. Treatment with pred significantly increased remission compared with treatment with AZA (OR, 0.21; 95\% CI, 0.06-0.71) and bude + AZA treatment significantly increased remission compared with placebo treatment 

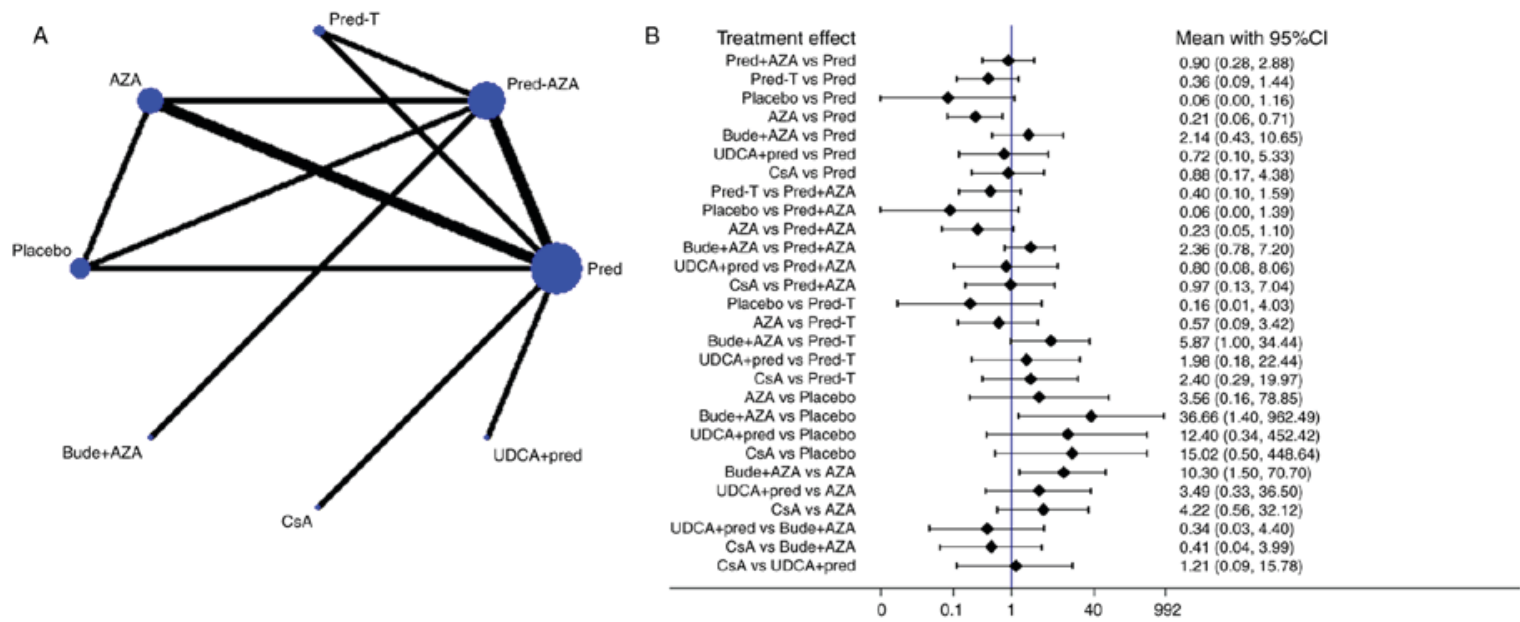

Figure 3. Network of treatment comparisons for remission. (A) Network plot indicating direct and indirect comparisons. The size of the nodes indicates the total sample size of treatments. The thickness of the line represents the number of trials that compare with each other. (B) Forest plot of network analysis. The network meta-analysis was calculated using the random effects model. Pred, prednisone; AZA, azathioprine; pred-T, prednisone in titrated doses given on alternate days; bude, budesonide; UDCA, ursodeoxycholic acid; CsA, cyclosporine-A; CI, confidence interval.

A
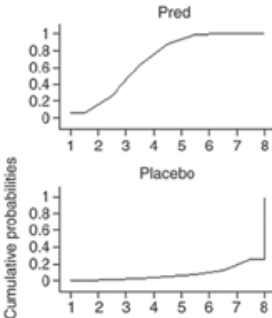

UDCA+Pred

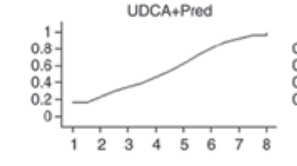

Graphs by treatment

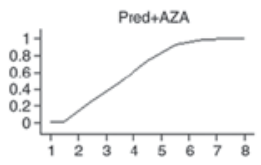

AZA

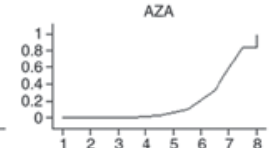

CsA

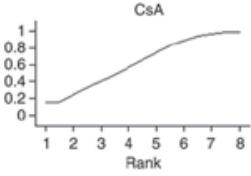

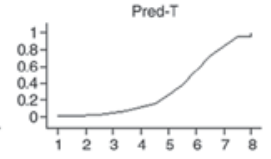

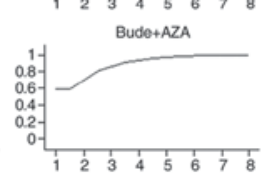

B Loop

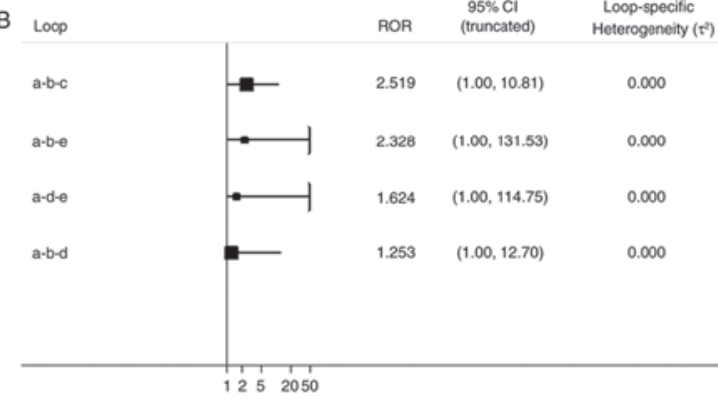

... Loop(s) [b-d-e] are formed only by multi-arm trialss) - Consistent by definition

Figure 4. Ranking of treatment strategies. (A) Ranking of treatment strategies based on the probability of their effects on outcome of remission. (B) Inconsistency plot for primary efficacy outcome of remission. Pred, prednisone; AZA, azathioprine; pred-T, prednisone in titrated doses given on alternate days; bude, budesonide; UDCA, ursodeoxycholic acid; CsA, cyclosporine-A; CI, confidence interval; a, pred; b, prednisone + AZA; c, pred-T; d, AZA; e, placebo; RoR, ratio of two odds ratios.

(OR, 36.66; 95\% CI, 1.40-962.49) or AZA (OR, 10.30; 95\% CI, 1.50-70.70). A ranking graph of distribution of probabilities on remission is presented in Fig. 4A. Based on SUCRA, bude + AZA treatment (89.4) was ranked first, pred (69.1) was ranked second, pred + AZA (63.2) and CsA (62.8) were ranked fourth and placebo was ranked last (7.8; Table III).

Inconsistency test. Inconsistency refers to differences between direct and various indirect effect estimates for the same comparison. The ratio of two odds ratios (RoR) from direct and indirect evidence in each loop was calculated to estimate the inconsistency. RoR values close to 1 mean that the direct and indirect evidence were in agreement. Fig. 4B demonstrated that in a total of four loops there were none with statistically significant inconsistency as all CIs for RoRs were compatible with zero inconsistency $(\mathrm{RoR}=1)$. These results indicate that direct and indirect estimates were consistent with one another.

Publication biases. Publication bias was not assessed because the number of RCTs was limited $(<10)$.
Other outcomes. The frequencies and percentages of adverse events were not recorded in the majority of articles (Table IV). Adverse events associated with pred and bude treatment were as follows: Cushingoid appearances, including moon face, acne and hirsutism; diabetes mellitus; hypertension; and cataracts. The adverse events following AZA therapy were gastrointestinal bleeding, leucopenia, trombopenia and arthralgia. Combination therapy with pred + AZA exhibited a markedly lower reported frequency of adverse events compared with pred monotherapy (data not shown). In addition, bude treatment had less adverse events compared with pred treatment (data not shown).

Table IV revealed the mortalities for all included studies. The direct comparisons on mortality were presented in Table V. The results indicated that patients treated with pred had significantly decreased rates of mortality compared with patients treated with the placebo (OR, 0.14; 95\% CI, 0.04-0.52). No significant differences in the mortality rates were identified between patients treated with pred and those treated with pred-T (OR, 1.61; 95\% CI, 0.25-10.40), AZA (OR, 0.29; 95\% 


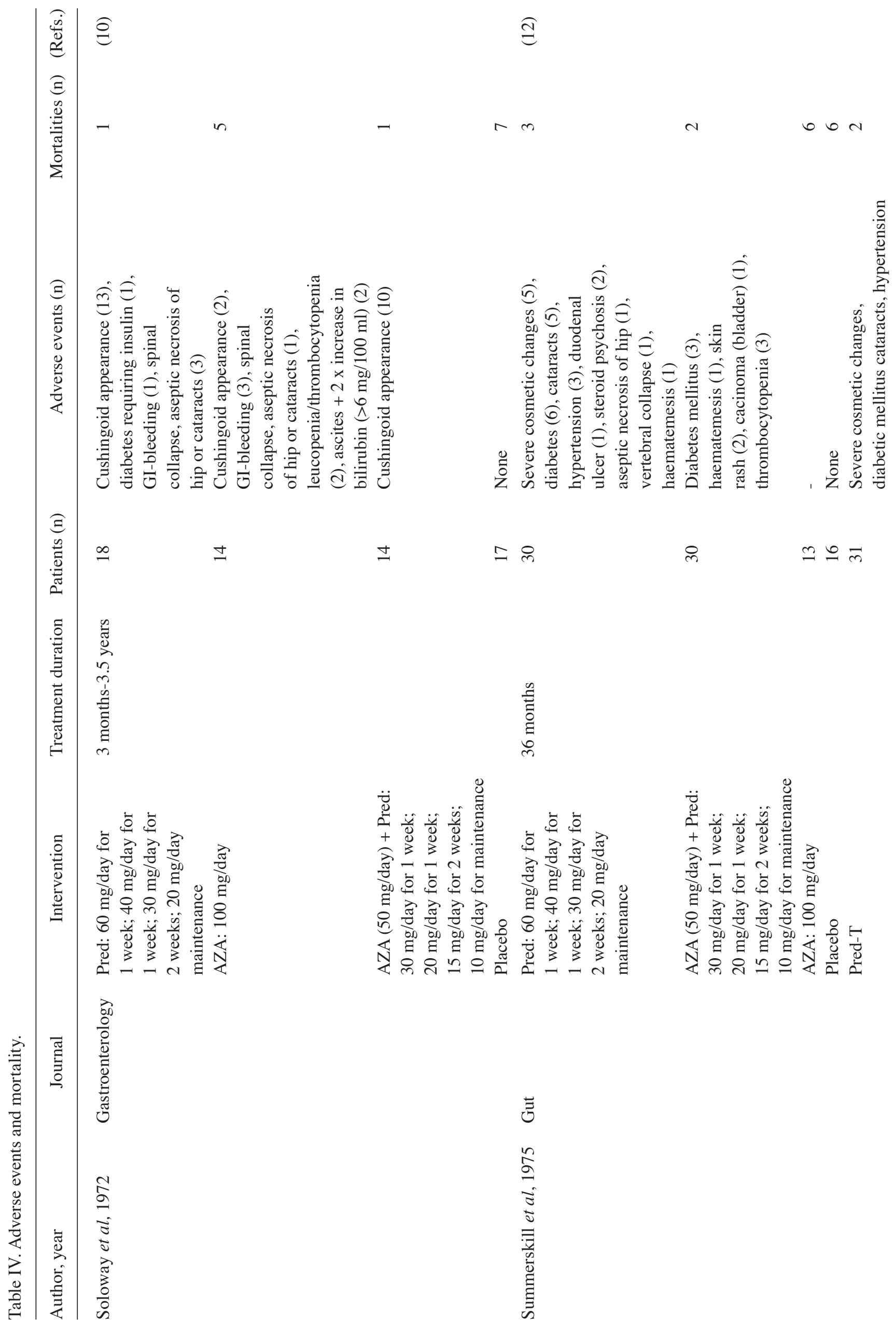




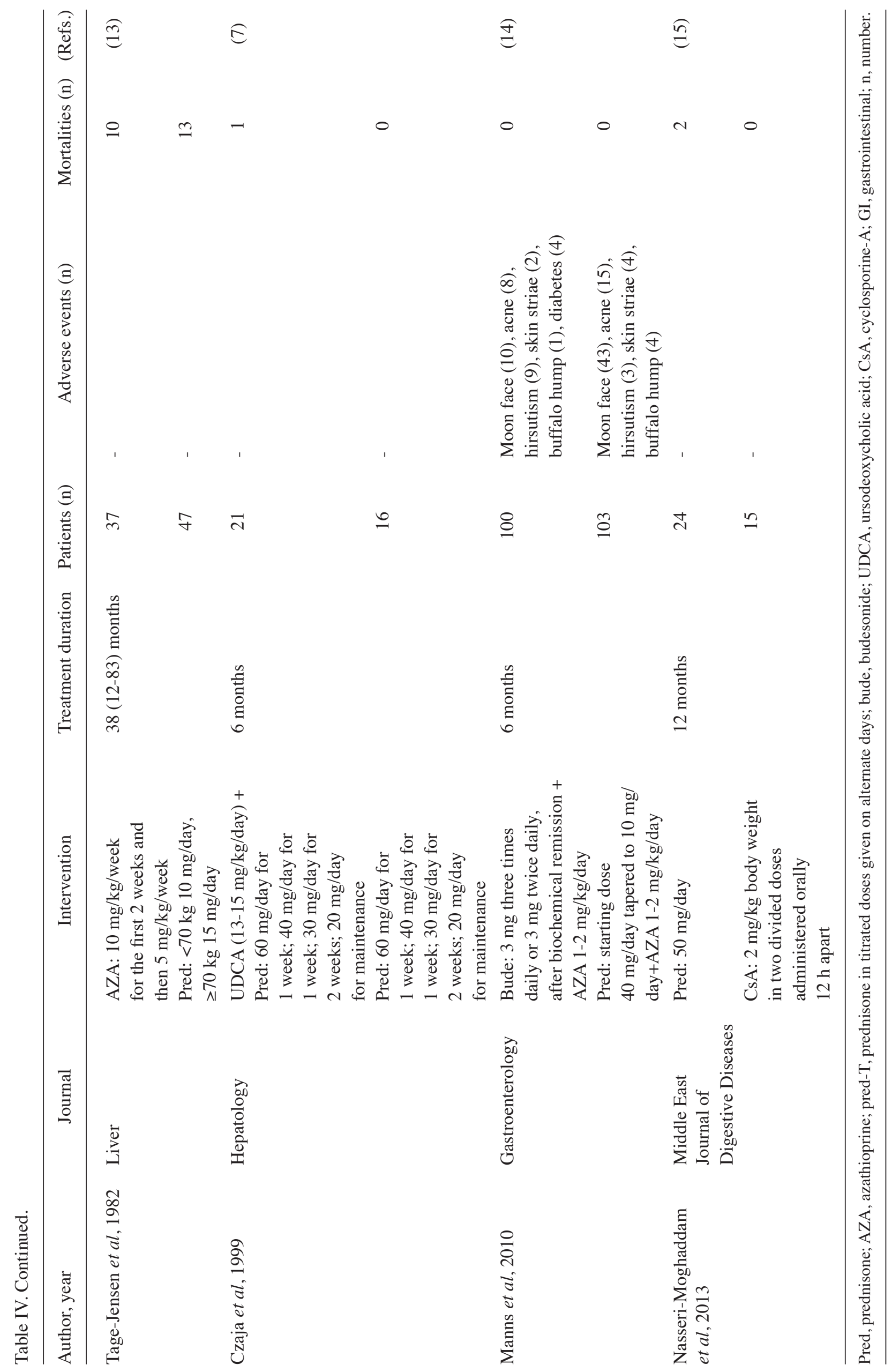


Table V. Mortality rates of different treatments.

\begin{tabular}{|c|c|c|c|c|c|c|}
\hline Treatment & Pred-T & Placebo & AZA & Bude + AZA & UDCA + Pred & CsA \\
\hline Pred & $1.61(0.25,10.40)$ & $0.14(0.04,0.52)^{\mathrm{a}}$ & $0.29(0.06,1.51)$ & - & $0.41(0.02,10.85)$ & $3.44(0.15,76.81)$ \\
\hline Pred + AZA & $1.04(0.14,7.87)$ & $0.12(0.03,0.46)^{\mathrm{a}}$ & $0.10(0.02,0.42)^{\mathrm{a}}$ & $0.97(0.06,15.74)$ & - & - \\
\hline
\end{tabular}

For mortality rates, data as presented as the odds ratio (95\% confidence interval) was calculated. If the OR and $95 \%$ CI were not close to 1 ,

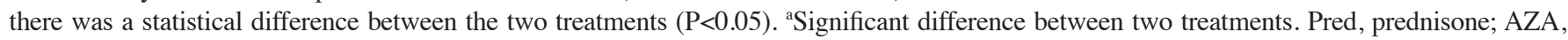
azathioprine; pred-T, prednisone in titrated doses given on alternate days; bude, budesonide; UDCA, ursodeoxycholic acid; CsA, cyclosporine-A.

CI, 0.06-1.51), UDCA + pred (OR, 0.41; 95\% CI, 0.02-10.85) and CsA (OR, 3.44; 95\% CI, 0.15-76.81). In addition, patients treated with pred + AZA had significantly decreased rates of mortality compared with patients treated with the placebo (OR, 0.12; 95\% CI, 0.03-0.46) and AZA (OR, 0.10; 95\% CI, $0.02-0.42)$. No significant differences in the mortality rates were identified between patients treated with pred + AZA and those treated with and pred-T (OR, 1.04; 95\% CI, 0.14-7.87), bude + AZA (OR, 0.97; 95\% CI, 0.06-15.74). No statistical differences of mortality rate were identified between AZA therapy and placebo treatment (data not shown).

\section{Discussion}

The network meta-analysis performed in the present study provided hierarchies of treatments for clinical and biochemical remission in adult patients with AIH. The meta-analysis demonstrated that the remission of patients was significantly increased following treatment with pred compared with AZA and was significantly increased following treatment with bude + AZA compared with placebo or AZA. Direct comparison demonstrated that remission of patients was significantly increased following treatment with pred compared with AZA or placebo treatment and similarly, remission of patients was significantly increased following treatment with pred + AZA compared with pred-T treatment. In addition, direct comparison indicated that patient remission was significantly increased following treatment with bude + AZA compared with pred + AZA. Treatments were ranked for increasing remission as follows: Bude + AZA, pred, pred + AZA or CsA, UDCA + pred, pred-T, AZA and placebo. Direct comparisons indicated that the frequency of adverse events in patients treated with pred + AZA combination therapy was decreased compared with that in patients treated with pred monotherapy, and patients treated with bude experienced fewer adverse events compared with those treated with pred alone. Furthermore, direct comparisons also demonstrated that the mortality of patients who underwent AZA or placebo treatment was increased compared with that of patients who underwent treatment with pred or pred + AZA; however, there was no notable difference between other treatments and pred or pred + AZA treatment.

The present study has a range of strengths: i) The search strategy was comprehensive in order to decrease the possibilities of publication bias; ii) only RCTs without high risk of bias were included in the current study; iii) network meta-analysis was used in the current study, which produces direct and indirect evidence in a network of trials that compare multiple interventions; and iv) the eight treatments included in the study were ranked based on the posterior probabilities of outcomes and SUCRA.

However, there are also limitations to the current study. The results were affected by study characteristics, including sex, detection bias and performance bias. In addition, the primary outcome measures did not include histological remission and may have an impact on the assessment of treatment efficacy, and an evolving set of diagnostic criteria may affect the objectivity of the results.

Pred alone or in combination with AZA was demonstrated to be more effective compared with AZA or placebo monotherapy in early studies, and therefore has become the standard therapy used to treat patients with AIH (35). Furthermore, treatment with pred + AZA had fewer adverse events compared with pred treatment alone $(35,36)$, which was consistent with the results of the current study. Bude therapy in combination with AZA has now emerged as an alternative frontline therapy for classical therapy in AIH (37-39). Through random experiments, Woynarowski et al (24) also discovered that bude may be an alternative to standard pred therapy in children. However, Czaja and Lindor (40) demonstrated that bude therapy had a low frequency of remission in ten treatment-dependent patients with AIH. Peiseler et al (41) also demonstrated that the efficacy of bude therapy may be lower compared with that of pred therapy alone; however, this effect may have been caused by the different study populations used. Danielsson and Prytz (42) indicated that oral bude therapy decreased liver inflammation in patients with non-cirrhotic AIH only. The network analysis in the current study ranked bude + AZA as higher than pred + AZA. Furthermore, descriptive analysis indicated that patients undergoing bude treatment experienced fewer adverse events compared with those who underwent pred treatment alone. The study population of included RCTs focusing on bude + AZA treatment was patients with non-cirrhotic AIH, and other studies did not distinguish between cirrhotic and non-cirrhotic patients. Therefore, further large-scale studies with a longer duration of follow-up history and a focus on dose response are required to investigate the use of bude + AZA as frontline therapy in adults with AIH. However, the results of the current study suggest that bude + AZA may be the most appropriate candidate for treatment of non-cirrhotic patients, which is consistent with the results demonstrated by Czaja (43).

The results of the current study demonstrated that treatment with CsA, UDCA + pred or pred-T resulted in increased 
remission rates compared with that observed in patients who received placebo treatment. Treatment with CsA, UDCA + pred or pred-T also had the same mortality rate as standard therapy. Thus, these therapies may be used as alternative treatment in patients with $\mathrm{AIH}$ who cannot undergo classical therapy. No statistical differences of mortality rate were identified between AZA therapy and placebo treatment; therefore, AZA is not recommended for treatment of patients with AIH, which is consistent with the conclusions provided by Gleeson (35).

It has been demonstrated that mycophenolate mofetil (MMF) (5,44-51), methotrexate (52), 6-mercaptopurine (53), allopurinol $(54,55)$, tacrolimus $(8,45,56-59)$ and everolimus (60) are effective and well-tolerated in patients with AIH. Torisu et al (61) indicated that UDCA monotherapy may be considered for treatment of patients with AIH with a serum ALT level of $<200 \mathrm{IU} / 1$. Among these treatments, MMF and tacrolimus were the most studied. Efe et al (62) also demonstrated that tacrolimus was more effective compared with MMF as therapy for difficult-to-treat AIH. In addition, MMF (63), calcineurin inhibitors $(64,65)$, sirolimus $(66,67)$, denosumab (68), rituximab (69) and infliximab (70) have been used successfully as salvage therapies in small observational studies. Although these therapies have been effective as treatment for patients with AIH, their superiority to standard treatment has not been evaluated. Therefore, clinical RCTs are required. Cytotoxic T lymphocyte antigen-4 (71), non-mitogenic antibodies to cluster of differentiation 3 (72), regulatory $\mathrm{T}$ cell promoters $(73,74)$, natural killer T cell modulators $(75)$, anti-fibrotic agents (76), monoclonal antibodies or nanobodies to chemokines (77) and anti-apoptotic agents (78) have been proposed for the treatment of autoimmune disease and may be novel alternative treatments for $\mathrm{AIH}$; however, this requires further study.

In conclusion, the results of the present study based on relatively small numbers suggest that treatment with pred alone or in combination with AZA remain the standard treatment for AIH. Bude in combination with AZA may be the most appropriate treatment for non-cirrhotic patients. However, bude + AZA as frontline therapy in adults with AIH requires additional large-scale studies with a longer duration of follow-up histology and a focus on dose-response. Additionally, development of other prospective treatments, which may be used as alternative therapies or first line therapies, and their subsequent evaluation in clinical RCTs is required.

\section{Acknowledgements}

The authors wish to thank members of Wenzhou Key Laboratory of Hepatology and the Hepatology Institute of Wenzhou Medical University for technical assistance. The present study was supported by the National Natural Science Foundation of China (grant nos. 81570514 and 81500477); Natural Science Foundation of Zhejiang Province (grant nos. LY15H030017 and LQ15H030006); Public Welfare Science and Technology Project of Wenzhou (grant no. Y20150014); Medical Award Fund (Beijing, China; grant no. YJHYXKYJJ-162); Scientific and Technological Innovation Team of the Early Warning and Intervention to End-stage Liver Disease of Wenzhou (grant no. C20150005); and the National Major Scientific and
Technological Special Project during the Twelfth Five-year Plan Period (China; grant nos. 2013ZX10005002-001-008, 2013ZX10002003 and 2012ZX10002004).

\section{References}

1. Manns MP, Czaja AJ, Gorham JD, Krawitt EL, Mieli-Vergani G, Vergani D and Vierling JM; American Association for the Study of Liver Diseases: Diagnosis and management of autoimmune hepatitis. Hepatology 51: 2193-2213, 2010.

2. Heneghan MA, Yeoman AD, Verma S, Smith AD and Longhi MS: Autoimmune hepatitis. Lancet 382: 1433-1444, 2013.

3. Gleeson D and Heneghan MA; British Society of Gastroenterology: British Society of Gastroenterology (BSG) guidelines for management of autoimmune hepatitis. Gut 60: 1611-1629, 2011.

4. Selvarajah V, Montano-Loza AJ and Czaja AJ: Systematic review: Managing suboptimal treatment responses in autoimmune hepatitis with conventional and nonstandard drugs. Aliment Pharmacol Ther 36: 691-707, 2012.

5. Zachou K, Gatselis N, Papadamou G, Rigopoulou EI and Dalekos GN: Mycophenolate for the treatment of autoimmune hepatitis: Prospective assessment of its efficacy and safety for induction and maintenance of remission in a large cohort of treatment-naïve patients. J Hepatol 55: 636-646, 2011.

6. Czaja AJ, Wang KK, Shiels MT and Katzmann JA: Oral pulse prednisone therapy after relapse of severe autoimmune chronic active hepatitis. A prospective randomized treatment trial evaluating clinical, biochemical, and lymphocyte subset responses. J Hepatol 17: 180-186, 1993.

7. Czaja AJ, Carpenter HA and Lindor KD: Ursodeoxycholic acid as adjunctive therapy for problematic type 1 autoimmune hepatitis: A randomized placebo-controlled treatment trial. Hepatology 30: 1381-1386, 1999.

8. Van Thiel DH, Wright H, Carroll P, Abu-Elmagd K, Rodriguez-Rilo H, McMichael J, Irish W and Starzl TE: Tacrolimus: A potential new treatment for autoimmune chronic active hepatitis: Results of an open-label preliminary trial. Am J Gastroenterol 90: 771-776, 1995.

9. Cook GC, Mulligan R and Sherlock S: Controlled prospective trial of corticosteroid therapy in active chronic hepatitis. Q J Med 40: 159-185, 1971.

10. Soloway RD, Summerskill WH, Baggenstoss AH, Geall MG, Gitnićk GL, Elveback IR and Schoenfield LJ: Clinical, biochemical, and histological remission of severe chronic active liver disease: A controlled study of treatments and early prognosis. Gastroenterology 63: 820-833, 1972 .

11. Murray-Lyon IM, Stern RB and Williams R: Controlled trial of prednisone and azathioprine in active chronic hepatitis. Lancet 1: 735-737, 1973.

12. Summerskill WH, Korman MG, Ammon HV and Baggenstoss AH: Prednisone for chronic active liver disease: Dose titration, standard dose, and combination with azathioprine compared. Gut 16: 876-883, 1975.

13. Tage-Jensen U, Schlichting P, Aldershvile J, Andersen P, Dietrichson O, Hardt F, Mathiesen LR and Nielsen JO: Azathioprine versus prednisone in non-alcoholic chronic liver disease (CLD). Relation to a serological classification. Liver 2: 95-103, 1982.

14. Manns MP, Woynarowski M, Kreisel W, Lurie Y, Rust C, Zuckerman E, Bahr MJ, Günther R, Hultcrantz RW, Spengler U, et al: Budesonide induces remission more effectively than prednisone in a controlled trial of patients with autoimmune hepatitis. Gastroenterology 139: 1198-1206, 2010.

15. Nasseri-Moghaddam S, Nikfam S, Karimian S, Khashayar P and Malekzadeh R: Cyclosporine-a versus prednisolone for induction of remission in auto-immune hepatitis: Interim analysis report of a randomized controlled trial. Middle East J Dig Dis 5: 193-200, 2013.

16. Boberg KM, Aadland E, Jahnsen J, Raknerud N, Stiris M and Bell $\mathrm{H}$ : Incidence and prevalence of primary biliary cirrhosis, primary sclerosing cholangitis, and autoimmune hepatitis in a Norwegian population. Scand J Gastroenterol 33: 99-103, 1998.

17. Werner M, Prytz H, Ohlsson B, Almer S, Björnsson E, Bergquist A, Wallerstedt S, Sandberg-Gertzén H, Hultcrantz R, Sangfelt $\mathrm{P}$, et al: Epidemiology and the initial presentation of autoimmune hepatitis in Sweden: A nationwide study. Scand J Gastroenterol 43: 1232-1240, 2008. 
18. Caldwell DM, Ades AE and Higgins JP: Simultaneous comparison of multiple treatments: Combining direct and indirect evidence. BMJ 331: 897-900, 2005.

19. Johnson PJ and Mcfarlane IG: Meeting report: International Autoimmune Hepatitis Group. Hepatology 18: 998-1005, 1993.

20. Higgins JP, Altman DG, Gøtzsche PC, Jüni P, Moher D, Oxman AD, Savovic J, Schulz KF, Weeks L and Sterne JA; Cochrane Bias Methods Group; Cochrane Statistical Methods Group: The cochrane collaboration's tool for assessing risk of bias in randomised trials. BMJ 343: d5928, 2011.

21. Chaimani A, Higgins JP, Mavridis D, Spyridonos P and Salanti G: Graphical tools for network meta-analysis in STATA. PLoS One 8: e76654, 2013.

22. Chen LX, Li YL, Ning GZ, Li Y, Wu QL, Guo JX, Shi HY, Wang XB, Zhou Y and Feng SQ: Comparative efficacy and tolerability of three treatments in old people with osteoporotic vertebral compression fracture: A network meta-analysis and systematic review. PLoS One 10: e0123153, 2015.

23. Sogo T, Fujisawa T, Inui A, Komatsu H, Etani Y, Tajiri H, Waki K, Shimizu Y, Nakashima S, Imagawa T and Yokota S: Intravenous methylprednisolone pulse therapy for children with autoimmune hepatitis. Hepatol Res 34: 187-192, 2006.

24. Woynarowski M, Nemeth A, Baruch Y, Koletzko S, Melter M, Rodeck B, Strassburg CP, Pröls M, Woźniak M and Manns MP; European Autoimmune Hepatitis-Budesonide Study Group: Budesonide versus prednisone with azathioprine for the treatment of autoimmune hepatitis in children and adolescents. J Pediatr 163: 1347-1353.e1, 2013.

25. Cuarterolo M, Ciocca M, López S, López V, Araujo M and Alvarez F: Initial treatment of autoimmune hepatitis in children: Neoral cyclosporine versus prednisone plus azathioprine. J Pediat Gastroenterol Nutr 63: S56, 2016.

26. Waisbourd-Zinman O, Hilmara D, Lin HC and Rand E: Steroid free treatment of autoimmune hepatitis in selected children. Hepatol 63: 354A-355A, 2016.

27. Stern RB, Wilkinson SP, Howorth PJ and Williams R: Controlled trial of synthetic D-penicillamine and prednisone in maintenance therapy for active chronic hepatitis. Gut 18: 19-22, 1977.

28. Hegarty JE, Nouri Aria KT, Eddleston AL and Williams R: Controlled trial of a thymic hormone extract (Thymostimulin) in 'autoimmune' chronic active hepatitis Gut 25: 279-283, 1984.

29. Stellon AJ,Hegarty JE, Portmann B and Williams R: Randomised controlled trial of azathioprine withdrawal in autoimmune chronic active hepatitis. Lancet 1: 668-670, 1985.

30. Stellon AJ, Keating JJ, Johnson PJ, McFarlane IG and Williams R Maintenance of remission in autoimmune chronic active hepatitis with azathioprine after corticosteroid withdrawal. Hepatology 8 : 781-784, 1988

31. Mucenic M, Mello ES and Cançado EL: Chloroquine for the maintenance of remission of autoimmune hepatitis: Results of a pilot study. Arq Gastroenterol 42: 249-255, 2005

32. Chi XL, Xiao HM, Xie YB, Cai GS, Jiang JM, Tian GJ, Shi MJ, Wu SD, Zhao PT and Chen HJ: Protocol of a prospective study for the combination treatment of Shu-Gan-jian-Pi decoction and steroid standard therapy in autoimmune hepatitis patients. BMC Complement Altern Med 16: 505, 2016.

33. Cai X, Weng HH and Miao LY: Yinzhihuang injection for treatment of patients with autoimmune hepatitis: Clinical efficacy and impact on hepatic fibrosis indexes. World Chin J Digestol 25: 726-731, 2017.

34. Imanieh MH, Khatami G and Ghavanini AA: Comparison of prednisolone alone and in combination with azathioprine regimens in treatment of autoimmune hepatitis: A prospective study. Iranian J Med Sci 25: 67-71, 2000.

35. Gleeson D: Standard treatment in adults: Which steroid? Or without steroids? Digest Dis (Basel, Switzerland) 33 (Suppl 2): S75-S82, 2015

36. Cropley A and Weltman M: The use of immunosuppression in autoimmune hepatitis: A current literature review. Clin Mol Hepatol 23: 22-26, 2017.

37. Czaja AJ: Current and future treatments of autoimmune hepatitis. Expert Rev Gastroenterol Hepatol 3: 269-291, 2009.

38. Czaja AJ: Drug choices in autoimmune hepatitis: Part A-steroids. Expert Rev Gastroenterol Hepatol 6: 603-615, 2012.

39. Czaja AJ: Nonstandard drugs and feasible new interventions for autoimmune hepatitis: Part I. Inflamm Allergy Drug Targets 11: 337-350, 2012

40. Czaja AJ and Lindor KD: Failure of budesonide in a pilot study of treatment-dependent autoimmune hepatitis. Gastroenterology 119: 1312-1316, 2000.
41. Peiseler M, Liebscher T, Pannicke N, Sebode M, Zenouzi R, Hartl J, Ehlken H, Weiler-Normann C, Lohse AW and Schramm C: Budesonide for autoimmune hepatitis: Response rate and limitations in a large real life cohort. J hepatol 62 (Suppl 2): S233, 2015.

42. Danielsson A and Prytz H: Oral budesonide for treatment of autoimmune chronic active hepatitis. Aliment Pharmacol Ther 8: 585-590, 1994

43. Czaja AJ: Current and prospective pharmacotherapy for autoimmune hepatitis. Expert Opin Pharmacother 15: 1715-1736, 2014.

44. Anderka MT, Lin AE Abuelo DN, Mitchell AA and Rasmussen SA: Reviewing the evidence for mycophenolate mofetil as a new teratogen: Case report and review of the literature. Am J Med Genet A 149A: 1241-1248, 2009.

45. Efe C, Hagström H, Bhanji RA, Müller NF, Wang Q, Purnak T, Muratori L, Werner M, Marschall HU, Muratori P, et al: Tacrolimus or mycophenylate mofetil as a second-line therapeutic options in patients with Autoimmune Hepatitis: An international multicentre observational study. AASLD 62: 109552, 2015

46. Zachou K, Gatselis N, Gabeta S, Saitis A, Koukoulis G and Dalekos GN: P1138: Long-term outcome of patients with autoimmune hepatitis receiving mycophenolate mofetil (MMF) as first line treatment. J Hepatol 62 (Suppl 2): S778-S779, 2015.

47. Gazzola A, Lim R, Strasser S, Nicoll A, Mitchell J, Siow W, Khoo T, Hamarneh Z, Weltman M, Janko N, et al: Mycophenolate in autoimmune hepatitis not responsive or intolerant to standard therapy: The TAPESTRY study. J Gastroenterol Hepatol (Australia) 31: 100-101, 2016.

48. Gazzola A, Lim R, Strasser SI, Nicoll A, Mitchell J, Siow W, Khoo TS, Hamarneh Z, Weltman M, Janko N, et al: Mycophenolate mofetil in autoimmune hepatitis patients not responsive or intolerant to standard therapy: The Australian tapestry study. Hepatology 63: 817A, 2016.

49. Kostyrko O, Shumilov P and Shigoleva N: Mycophenolate mofetil for the treatment of autoimmune hepatitis in children. J Hepatol 64: S640, 2016.

50. Park SW, Um SH, Lee HA, Kim SH, Sim Y, Yim SY, Seo YS and Ryu HS: Mycophenolate mofetil as an alternative treatment for autoimmune hepatitis. Clin Mol Hepatol 22: 281-285, 2016.

51. Zachou K, Gatselis NK, Arvaniti P, Gabeta S, Rigopoulou EI, Koukoulis GK and Dalekos GN: A real-world study focused on the long-term efficacy of mycophenolate mofetil as first-line treatment of autoimmune hepatitis. Aliment Pharmacol Ther 43 : 1035-1047, 2016.

52. Haridy J, Nicoll A and Sood S: Methotrexate therapy for autoimmune hepatitis. Clin Gastroenterol Hepatol: Jul 12, 2017 (Epub ahead of print)

53. Hübener S, Oo YH, Than NN, Hübener P, Weiler-Normann C, Lohse AW and Schramm C: Efficacy of 6-mercaptopurine as second-line treatment for patients with autoimmune hepatitis and azathioprine intolerance. Clin Gastroenterol Hepatol 14: 445-453, 2016.

54. Deswal S and Srivastava A: Role of allopurinol in optimizing thiopurine therapy in patients with autoimmune hepatitis: A review. J Clin Exp Hepatol 7: 55-62, 2017.

55. Terrabuio DR, De Moraes Falcao LT, Ono SK, Diniz MA, Carrilho FJ and Cancado E: Allopurinol is safe and effective to achieve biochemical and histological remission in patients with autoimmune hepatitis with incomplete therapeutic response. J Hepatol 64 (Suppl): S435, 2016.

56. Marlaka JR, Papadogiannakis N, Fischler B, Casswall TH, Beijer E and Németh A: Tacrolimus without or with the addition of conventional immunosuppressive treatment in juvenile autoimmune hepatitis. Acta Paediatr 101: 993-999, 2012.

57. Than NN, Wiegard C, Mann J, Fussel K, Hirschfield G, Lohse AW, Adams D, Schramm C and Oo YH: Tacrolimus is safe and effective in patients with resistant type 1 autoimmune hepatitis. J Hepatol 62: S805-S806, 2015.

58. Than NN, Wiegard C, Weiler-Normann C, Weiler-Normann C, Füssel K, Mann J, Hodson J, Hirschfield GM, Lohse AW, Adams DH, et al: Long-term follow-up of patients with difficult to treat type 1 autoimmune hepatitis on Tacrolimus therapy. Scand J Gastroenterol 51: 329-336, 2016.

59. Al Taii H, Hanouneh MA, Hanouneh I, Lopez R, Zein N and Alkhouri N: The use of tacrolimus in refractory autoimmune hepatitis in children and adults: A single center experience. Scand J Gastroenterol 52: 157-158, 2017.

60. Ytting $\mathrm{H}$ and Larsen FS: Everolimus treatment for patients with autoimmune hepatitis and poor response to standard therapy and drug alternatives in use. Scand J Gastroenterol 50: 1025-1031, 2015 
61. Torisu Y, Nakano M, Takano K, Nakagawa R, Saeki C, Hokari A Ishikawa T, Saruta M and Zeniya M: Clinical usefulness of ursodeoxycholic acid for Japanese patients with autoimmune hepatitis. World J Hepatol 9: 57-63, 2017.

62. Efe C, Hagstrom H, Ytting H, Bhanji RA, Müller NF, Wang Q, Purnak T, Muratori L, Werner M, Marschall HU, et al: Efficacy and Safety of Mycophenolate Mofetil and Tacrolimus as Secondline Therapy for Patients With Autoimmune Hepatitis. Clin Gastroenterol Hepatol 15: 1950-1956, 2017.

63. Baven-Pronk AM, Coenraad MJ, van Buuren HR, de Man RA, van Erpecum KJ, Lamers MM, Drenth JP, van den Berg AP, Beuers UH, den Ouden J, et al: The role of mycophenolate mofetil in the management of autoimmune hepatitis and overlap syndromes. Aliment Pharmacol Ther 34: 335-343, 2011.

64. Czaja AJ: Autoimmune hepatitis: Focusing on treatments other than steroids. Can J Gastroenterol 26: 615-620, 2012.

65. Czaja AJ: Drug choices in autoimmune hepatitis: Part B-Nonsteroids. Expert Rev Gastroenterol Hepatol 6: 617-635, 2012.

66. Kurowski J, Melin-Aldana H, Bass L, Alonso EM and Ekong UD: Sirolimus as rescue therapy in pediatric autoimmune hepatitis. J Pediatr Gastroenterol Nutr 58: e4-e6, 2014.

67. Chatrath H, Allen L and Boyer TD: Use of sirolimus in the treatment of refractory autoimmune hepatitis. Am J Med 127: $1128-1131,2014$

68. Ikejima K, Yaginuma R, Kon K, Aoyama T, Uchiyama A, Yamashina S and Watanabe S: Efficacy of denosumab on progression of osteoporosis in autoimmune liver diseases. Hepatol (Baltimore, Md) 63: 201A, 2016.

69. Gautam N, Than NN, Nizamuddin M, Adams D and Oo YH PTU-123 use of rituximab in resistant autoimmune hepatitis-Birmingham experience. Gut 63: A93, 2014

70. Weiler-Normann C, Schramm C, Quaas A, Wiegard C, Glaubke C, Pannicke N, Möller S and Lohse AW: Infliximab as a rescue treatment in difficult-to-treat autoimmune hepatitis. J Hepatol 58: 529-534, 2013.
71. Dhirapong A, Yang GX, Nadler S, Zhang W, Tsuneyama K, Leung P, Knechtle S, Ansari AA, Coppel RL, Liu FT, et al: Therapeutic effect of cytotoxic T lymphocyte antigen 4/immunoglobulin on a murine model of primary biliary cirrhosis. Hepatology 57: 708-715, 2013.

72. Killestein J: Anti-CD3 monoclonal antibody in new-onset type 1 diabetes mellitus. N Engl J Med 347: 1116-1117, 2002.

73. Czaja AJ: Challenges in the diagnosis and management of autoimmune hepatitis. Can J Gastroenterol 27: 531-539, 2013.

74. Lapierre P, Béland K, Yang R and Alvarez F: Adoptive transfer of ex vivo expanded regulatory T cells in an autoimmune hepatitis murine model restores peripheral tolerance. Hepatology 57: 217-227, 2013.

75. Blumenfeld HJ, Tohn R, Haeryfar SM, Liu Y, Savage PB and Delovitch TL: Structure-guided design of an invariant natural killer $\mathrm{T}$ cell agonist for optimum protection from type 1 diabetes in non-obese diabetic mice. Clin Exp Immunol 166: 121-133, 2011.

76. Czaja AJ: The prevention and reversal of hepatic fibrosis in autoimmune hepatitis (Review). Aliment Pharmacol Ther 39: 385-406, 2014.

77. Yellin M, Paliienko I, Balanescu A, Ter-Vartanian S, Tseluyko V, Xu LA, Tao X, Cardarelli PM, Leblanc H, et al: A phase II, randomized, double-blind, placebo-controlled study evaluating the efficacy and safety of MDX-1100, a fully human anti-CXCL10 monoclonal antibody, in combination with methotrexate in patients with rheumatoid arthritis. Arthritis Rheum 64: 1730-1739, 2012

78. Anstee QM, Concas D, Kudo H, Levene A, Pollard J, Charlton P, Thomas HC, Thursz MR and Goldin RD: Impact of pan-caspase inhibition in animal models of established steatosis and non-alcoholic steatohepatitis. J Hepatol 53: 542-550, 2010 . 